\title{
Editorial: The Potential Role of Endoscopic Findings of WLI Followed by $M-N B I$ in Diagnosing GP SNADET
}

\author{
Hang Yang Bing $\mathrm{Hu}$ \\ Department of Gastroenterology, West China Hospital, Sichuan University, Chengdu, China
}

\section{Dear Editor,}

We read with interest the article by Akazawa et al. [1] in a previous issue of Digestion. Endoscopic findings of white-light imaging (WLI) and magnifying narrow-band imaging (M-NBI) were found significantly different between gastric phenotype (GP) and intestinal phenotype (IP) non-ampullary duodenal epithelial tumors (SNADETs), indicating the potential role of endoscopic diagnosis and differential diagnosis of SNADETs.

From the results of this interesting research, we find that the location and lobular/granular pattern of WLI and WOS, LBCs, and DIP of M-NBI were almost totally opposite between GP and IP. If diagnosis process can be carried out as WLI followed by M-NBI to first confirm an epithelial-derived SNADET, an inference can be made further that an elevated (0-I or IIa) SNADET located in the first portion with positive lobular/granular pattern under WLI and with positive DL and DIP and without LBCs under M-NBI is GP, and the possibility is nearly $100 \%$. The clinical value of this inference for GP-SNADE is similar to the positive regular arrangement of collecting venules, indicating negative Helicobacter pylori infection. In addition, a brief score system can also be established based on the endoscopic findings of WLI followed by M-NBI for preoperative endoscopic diagnosis and predicting the potential cancerous transformation risk via mucin phenotypes (GP), lesion size, and vessel plus surface classification system $[1,2]$.

\section{Conflict of Interest Statement}

Both authors declare there is no conflict of interest.

\section{Fundings Sources}

This study did not receive support from any funding agency.

\section{Author Contributions}

Hang Yang and Bing Hu conceived the study. Hang Yang drafted the article. Bing Hu reviewed the article. All authors approved the final version of this manuscript.

\footnotetext{
References

1 Akazawa $\mathrm{Y}$, Ueyama H, Tsuyama S, Ikeda A, Yatagai N, Komori H, et al. Endoscopic and clinicopathological features of superficial non-ampullary duodenal tumor based on the mucin phenotypes. Digestion. 2021;102(5): 663-70.

2 Tanaka Y, Fujii S, Oiwa Y, Kusaka T, Shibuya S, Kokuryu H. Efficacy of magnifying narrow band imaging with acetic acid spray in diagnosing superficial non-ampullary duodenal epithelial tumors. Digestion. 2021;102(4): 572-9.
}

karger@karger.com

www.karger.com/dig

Karger'
(C) 2022 S. Karger AG, Basel
Correspondence to:

Bing Hu, hubingnj@163.com 